\title{
Serious Games as a means for holistically supporting Parkinson's Disease patients: The i-PROGNOSIS Personalized Game Suite framework
}

\author{
S. B. Dias ${ }^{1}$, E. Konstantinidis ${ }^{2}$, J. A. Diniz 1 , P. Bamidis ${ }^{2}$, V. Charisis ${ }^{2}$, S. Hadjidimitriou ${ }^{2}$, M. Stadtschnitzer ${ }^{3}$, P. \\ Fagerberg $^{4}$, I. Ioakeimidis ${ }^{4}$, K. Dimitropoulos ${ }^{5}$, N. Grammalidis ${ }^{5}$, and L. J. Hadjileontiadis ${ }^{2,6}$ \\ ${ }^{1}$ Faculdade de Motricidade Humana, Universidade de Lisboa, Portugal, sbalula@fmh.ulisboa.pt, jadiniz@ fmh.ulisboa.pt \\ ${ }^{2}$ Aristotle University of Thessaloniki, Thessaloniki, Greece, evdokimosk@gmail.com, bamidis@med.auth.gr, \\ stellios22@gmail.com,vcharisis@ee.auth.gr, leontios@auth.gr \\ ${ }^{3}$ Fraunhofer Institute IAIS, Sankt Augustin, Germany, michael.stadtschnitzer@iais.fraunhofer.de \\ ${ }^{4}$ Karolinska Institutet, Stockholm, Sweden, petter.fagerberg@ki.se, Ioannis.Ioakimidis@ki.se \\ ${ }^{5}$ Centre for Research \& Technology, Hellas, Thessaloniki, Greece, ngramm@iti.gr \\ ${ }^{6}$ Khalifa University of Science and Technology, Abu Dhabi, P.O. BOX 127788, UAE, leontios.h@kustar.ac.ae
}

\begin{abstract}
Parkinson's Disease (PD) is a well-known progressive and neurodegenerative disorder, resulting in motor and non-motor symptoms that significantly reduce the quality of life. Motivated by the increased adoption of serious games in health care interventions sector in the last years, an unobtrusive approach is explored here, realised via the i-PROGNOSIS interventions platform (www.i-prognosis.eu), consisting of a game-based suite. In this way, targeting intelligent early detection and intervention in PD area, the Personalised Game Suite (PGS) design approach is presented, integrating different serious games in a unified platform. Overall, this comprehensive review gives an overview of studies examining motor and non-motor symptoms for PD along with the PGS design approach, in order to inform researchers and game designers for its consideration in the self-management of PD.
\end{abstract}

Keywords-Parkinson's Disease; i-PROGNOSIS; Personalized Game Suite; Serious Games

\section{INTRODUCTION}

$\mathrm{P}$ ARKINSON's Disease (PD) is considered a gradually progressing neurodegenerative disorder, characterized by a reduction of neurons in the substantia nigra, resulting in the range of debilitating motor and non-motor symptoms. Reflecting the opportunities of Serious Games (SGs) in the field of personal health, a systematic review of twenty-one serious games for health and education, described at scientific papers and 7th Framework Program Projects, is explored in [1]. In this way, motivated by the recent and increased adoption of SGs in health care interventions sector [2-5], an alternative approach is presented here, as part of the H2020 iPROGNOSIS project (www.i-prognosis.eu), consisting of a game-based suite, defined as Personalized Game Suite (PGS), in order to mitigate the PD symptoms in a personalized and gamified environment. Targeting intelligent early detection and intervention in PD area, the PGS design introduces the integration of different serious games in a unified platform, namely: ExerGames, DietaryGames, EmoGames, and Handwriting/Voice Games, targeting the PD symptoms. Moreover, in the i-PROGNOSIS intervention Platform, integrated technology modules will be developed to monitor and support older adult's physical and emotional status enhancement, towards the decrease of the PD-related risks and increase of their quality-adjusted life-years (QALYs).
The rest of the paper is structured as follows: first, a literature review related with interventions for PD, along with the evidence-based on SGs is presented, followed by the description of the proposed PGS design. Next, a discussion related with the evaluation of the games is provided. Finally, conclusions and future work conclude the paper.

\section{THE PGS BACKGROUND}

\section{A. Interventions for $P D$}

Multiple interventions related with specific PD symptoms have been explored in the last years to improve the quality of life of PD patients, categorized below:

Gait mechanisms: aerobic exercise has showed immediate beneficial effects in improving motor action, balance, and gait in patients with PD [6]; treadmill training associated with auditory and visual cues might give better results to PD patients than more conventional treatments [7]; altering distribution of body weight from leg to leg overcoming virtual obstacles changing direction while walking [8]; possibility of counting loudly or silently and taking steps with the rhythm progressive resistance exercise can improve gait speed and muscle strength [9]; music cuing for improving the performance of PD patients in a game-like environment [10].

Tremor, bradykinesia and limited range of motion (ROM): analyzing kinetic tremor using circle movements' Wacom tablet, so to produce a useful marker in evaluation of impact of Deep Brain Stimulation in PD patients [11]; integrating of "BIG" training to improve speed and amplitude [12].

Balance, coordination and abnormal posture: combining multi-mode exercise and adaptive programs to improve balance and mobility in the patients with PD [13]; provision of external focus instructions by carer to reduce postural instability in PD patients [14]; having twice weekly Tai Chi lessons for improvements in gait, balance and functional mobility [15]; combining power training and high-speed yoga programs to significantly improve physical performance (e.g., postural balance, muscle strength) in older persons with PD [16].

Meal mechanisms and daily meal distribution: following motor swallowing exercises to reduce prevalence of swallowing disorders and to improve self-reported quality of life among PD patients [17]; having a brief session of gum chewing to improve swallow frequency and latency acutely (at 
least 5 minutes after the chewing session), among PD patients [18]. Avoiding irregular meal patterns as they are pointed out as negative for cardiometabolic health [19], therefore, health benefits might be gained from adopting a more regular meal pattern with a meal frequency that fits the individuals' lifestyle.

Preferred food characteristics: the relevant interventions should consider that there are two main eating patterns of normal-weight individuals: decelerated and linear eating [20], with the former referring to a decreased eating rate as the meal reaches its end, and the later referring to meals when the individual retains a steady eating rhythm throughout the course of the meal; yet, clinical populations with obesity or eating disorders are characterized by linear eating [21]. For decelerated eaters $(\alpha<0)$, the initial Food Intake (FI) rate $\beta$ was typically higher than that of linear eaters ( $\alpha$ close to 0 ), however, the total meal FI does not differ significantly between normal-weight linear and decelerated eaters [20]. These findings combined, provide evidence for the relation of the linear eating style to the risk of developing disordered eating. Interestingly, the use of the Mandometer can train away the suspect behavior [20]. In this vein, new algorithms were proposed that automatically compute the FI curve solely based on Mandometer recordings, enabling the use of the Mandometer method/treatment in non-laboratory conditions, without the need for supervision [22], allowing for designing more accurate nutrition interventions

Dietary Quality: adopting higher adherence to a Mediterranean diet to achieve reduced risk of cognitive decline and essential tremor [23] as conditions related with neurodegeneration; meta-analysis of prospective cohort studies that involved 1574299 participants showed a significant reduction in overall mortality (9\%), mortality from cardiovascular diseases (9\%), incidence of or mortality cancer (6\%), and incidence of PD and Alzheimer's disease (13\%) when greater adherence to the Mediterranean diet was observed [24]; after 16 years of follow up, in the work of [25] it was found 508 new cases of PD, showing an association between adherence to the Mediterranean diet and risk of this disease. In fact, the subjects who were in the highest quintile of adherence to the Mediterranean diet score, showed a $25 \%$ reduction in the risk of PD in comparison to those who had a lower score of adherence; hence, adherence to a Mediterranean diet may be associated with a reduced risk of PD, whereas low adherence is associated with an earlier age of onset [26].

Non-motor symptoms, psychological issues, depression: interventions based on the recent advances in natural language processing, text analysis and computational linguistics that facilitate sentiment extraction from different text sources (e.g., social media, blogs, SMS); in the work of [27], a supervised machine learning approach was adopted, in order to recognize six basic emotions (i.e., anger, disgust, fear, happiness, sadness and surprise) using a heterogeneous emotion-annotated dataset which combines news headlines, fairy tales and blogs.

Hypomimia: automatically detecting and assessing the severity of hypomimia, using machine learning tools and a 3D sensor that allows for fairly accurate facial movements tracking [28]; using of ultrasonic markers placed on participants faces to investigate the hypomimia condition in patients suffering from depression [29].

Handwriting mechanisms: spiral drawing has been used for the assessment of the impact of therapy on motor performance in various movement disorders (including in PD), and words containing one or multiple repetitions of cursive letter "I" are often used to evaluate handwriting samples [30]. In this context, a two-week self-administrated handwriting therapeutic exercise protocol, called Amplified Air Writing (AAW), has been conducted by six PD subjects who have demonstrated micrographia symptoms, to investigate its influence on their writing samples. In addition, to study handwriting changes, several computerized metrics to quantify micrographia were implemented. Metric results demonstrated significant temporary improvement for post-AAW handwriting performance; cursive samples especially show clear benefits for the subjects [31]; simple parallel horizontal lines improved handwriting in people with PD who have a problem with micrographia [32]; the authors also demonstrated that the use of both parallel lines and grid lines improved the letter size of PD patients compared to free writing; in addition, the use of visual (dots indicating size) and verbal cues ('big') improved the writing of PD patients with a more normal amplitude [33].

Voice mechanisms: adopting traditional methods of speech therapy for dysarthric patients with PD, typically administered once or twice a week and emphasizing articulation, rate, and prosody intervention, has been largely ineffective. By contrast, implementing intensive voice therapy methods, administered almost daily and emphasizing simple phonatory effort tasks, have been found to produce favorable results [34], such as the Lee Silverman voice treatment program $\left(\mathrm{LSVT}^{\circledR}\right)$, which focuses on a simple set of tasks designed to maximize phonatory and respiratory functions [34].

Considering the aforementioned relevant literature review, there is still a gap in the understanding on how these types of interventions are used for the self-management of the PD patients, in a holistic and unobtrusive way. From this perspective, motivated by the increased adoption of SGs in health care interventions sector in the last years, an extensive literature review contextualized with the corresponding motor and non-motor symptoms for PD along with the evidence based on SGs is presented in the succeeding subsection.

\section{B. Serious-Games Interventions for PD}

As mentioned in previous sections, recent SG-based approaches targeting the main PD symptoms have been investigated in different fields, namely: a) evidence for exercise-based serious games (ExerGames): gait mechanics, presence of tremor, bradykinesia and limited range of motion, balance and coordination issues, abnormal posture and physical status; b) evidence for dietary habits-based serious games (DietaryGames): meal mechanics, daily meal distribution, preferred food characteristics, and dietary quality; c) evidence for emotional aspects-based SGs (EmoGames): non-motor symptoms, psychological issues, depression, and hypomimia; and d) evidence for handwriting-voice aspects-based serious games (Handwriting-Voice (H/V) Games): handwriting and voice mechanics (see Table I). 


\begin{tabular}{|c|c|}
\hline PD target Symptoms & EXERGAMES-related evidence based on SGs \\
\hline $\begin{array}{l}\text { Gait mechanics [8], } \\
{[35]}\end{array}$ & $\begin{array}{l}\text { External auditory, visual or tactile cues are established as non-pharmaceutical methods to overcome gait difficulties [36]; A collection } \\
\text { of five motion-based games for PD patients, aimed at supporting some types of exercises and routines in a playful environment is } \\
\text { described in [37]; however, in order to determine if the mechanics adopted can help PD patients to reduce physical symptoms, some } \\
\text { long-term evaluation studies are recommended. Aerobic exercise should be included to improve gait in PD patients [38]. }\end{array}$ \\
\hline Tremor [35] & $\begin{array}{l}\text { Some games are designed too complex and needed to be made easier for PD patients, in order to avoid difficulties with the hand-held } \\
\text { controllers [37]. Hand-held devices or remotes are not recommended [38]. }\end{array}$ \\
\hline Bradykinesia [35], [39] & $\begin{array}{l}\text { Drawing continuous circles can help to detect symptoms as a sign of bradykinesia [40]; concepts of accuracy and timing can help and } \\
\text { reduce symptoms related to bradykinesia [41]. For example, in a rowing game, participants rowed faster in the game when the audio } \\
\text { tempo was increased as the game progressed, since audio with beat tempo was simultaneously provided [42] }\end{array}$ \\
\hline $\begin{array}{l}\text { Limited Range of } \\
\text { Motion (ROM) [43] }\end{array}$ & $\begin{array}{l}\text { In the ROM-related SGs, an automatic or semi-automatic calibration system should be incorporated to match the ROM of the patient } \\
\text { with the ROM required by the virtual game player [42]; a calibration algorithm has to be employed by registering the maximum ROM } \\
\text { and matching it with the desirable ROM in the virtual environment [42]. In the latter, the individuals display extended movement } \\
\text { following initial phases of "warm up" or in accordance to the game level and excitement [42]. Moreover, dynamically calculated ROM } \\
\text { and the mapping of the movement to the virtual environment dynamically adjusted should be considered [42]. A calibration based on } \\
\text { three predefined poses can be performed to determine a suitable amplitude setting for the first game and to constrain dynamic } \\
\text { amplitude adaptation to a reasonable maximum [44]. As pointed out, required ROM steadily increases during the first game rounds } \\
\text { until the calibrated constraints are reached. On the one hand, the participants needed to fully stretch their arms and their back at the } \\
\text { high-level settings; on the other hand, the system needs to be flexible and allow for fine-grained control to encompass individual } \\
\text { strengths and weaknesses. }\end{array}$ \\
\hline $\begin{array}{l}\text { Balance and } \\
\text { coordination issues [45] }\end{array}$ & $\begin{array}{l}\text { Based on several structured clinical discussions, among others, multi-directional stepping exercises for PD patients are described as } \\
\text { important recommendations [46]. An 8-week exercise training class by using the Wii Fit balance board improved selective measures of } \\
\text { balance and gait in adults with PD [47]. In the work of [48], SGs were divided into three groups, namely: 1) static balance (Single Leg } \\
\text { Extension and Torso Twist); 2) dynamic balance (Table Tilt, Tilt City, Soccer Heading and Penguin Slide); and 3) stationary gait } \\
\text { (Rhythm Parade, Obstacle Course, Basic Step and Basic Run). The authors concluded that after } 14 \text { sessions of balance training, the PD } \\
\text { patients showed improved performance in activities of daily living [48]. }\end{array}$ \\
\hline $\begin{array}{l}\text { Abnormal posture [35], } \\
\text { [49] }\end{array}$ & $\begin{array}{l}\text { Training based on self-correcting one's posture with visual feedback and maintaining the posture while focusing attention on } \\
\text { proprioceptive and somatosensory feedback might correct misperceptions of the vertical position and improve standing posture in PD } \\
\text { patients [50]. Stretching can reduce the shortening of flexor muscles that contributes to the abnormally flexed posture and balance } \\
\text { exercise (alone or combined with other training modalities) can improve postural control [51]. In [52], a Kinect-based Unity3D } \\
\text { Exergame supports a collection of gestures drawn from existing PD-specific training programs which advocate big and purposeful } \\
\text { movements, in order to improve postural stability and the overall mobility of upper and lower limbs [52]. }\end{array}$ \\
\hline
\end{tabular}

\section{PD target Symptoms}

Meal mechanics: dysphagia [53]

Daily meal distribution: behavior [55]

Preferred food characteristics: preference to semi-solid diets [55], [57] Dietary Quality: constipation [58], [59]

\section{PD target Symptoms}

Psychological issues: depression, loss of selfconfidence, embarrassment, stress [62], [63] compulsive eating decrease of water intake

\section{DIETARYGAMES-related evidence based on SG}

A game-based assistive tool for rehabilitation of dysphonic patients was developed in [54], where the voice exercises embed in the developed game can not only improve the pronunciation, but also enhance the swallowing muscle of the patient, in case the patient accidentally eats the food into lung.

Efforts has been made to increase impulse control related to eating behavior, such as in [56], where an iOS app was developed to instruct users to tap images of "healthy" food as fast and accurate as possible, while refraining from tapping on images related to "unhealthy" food. However, further research is needed to assess if the observed changes transfer to longer-term real-life contexts (e.g., weight loss)

The preliminary study of [56] investigated the efficacy of training to improve inhibitory control (IC), known as a process connected with the overeating, so to affect users' food preferences and impulsive eating behavior, by using a multisession mobile phone-based intervention to train IC in an overweight and obese population. The results revealed that the IC training using the purpose-designed mobile phone app is easy to complete, convenient, and can increase cognitive restraint and reduce unhealthy food consumption. MyBehavior, a mobile phone app, was designed to process tracked eating behavior and physical activity data, in order to provide personalized, actionable, low-effort suggestions that are contextualized to the user's environment and previous behavior from a selftracked information (i.e., manual food logging) [60]. In order to foster healthy eating, a technology-enhanced approach, game-based team learning (GBTL), was proposed in [61], providing feedback on the nutritional intake of learners through a meal analysis algorithm, allowing teams to compete against each other on the basis of each group's dietary habits.

EMOGAMES-related evidence based on SGs the PD patients emphasized the physiological importance of practicing exercise in a regular way, allowing to self-manage the PD symptoms (e.g., stiffness, pain), as well as the importance of the inclusion of in-game notifications (e.g., score, progress, difficulty levels). Sound effects should be included in SGs, in order to encourage PD patients (e.g., clapping, whistle) [40], [46], as well as rewards when accomplishing a task (e.g., smiley faces, winning points, piece of exciting music) [40], [64]. For instance, when the player completes a task, a piece of exciting music should be used to start as an encouragement [40]; moreover, the players should also be rewarded during their effort and be encouraged to keep trying [37]. Understanding fields of interests/preferences and avoiding negative and violent contents are key aspects for the creation of appealing game-scenarios for elderly [37]. The design of the games should avoid an adventure or complex narrative-based game, especially science fiction [65], and adopt the idea of solo play over play with others (promoting self-conscious over performance). Some players enjoy trying to improve their personal-best ratings, while others are more interested in competing against each other [42]. Appealing graphics, attractive scenes, charming animations and amusing sounds are essential elements of fun and directly related to the engagement of a player [42].

Hypomimia [35] Several games challenge the player to [66]: observe gaze directions in facial images; match faces; reconstruct face sequence; memorize social details regarding sequentially presented faces; identify smiling faces; find faces whose expression matches the target; identify emotions implicitly expressed by facial expressions; match pairs of similar facial expressions; match sentences with tags that identify emotional prosodies with which they are expressed; identify social scenarios that best explain emotions expressed in video clips; choose expressions that best describe how a person would sound in given social scenarios, in order to understand/interpret, in a progressive way, more complex social environments [66] 


\begin{tabular}{ll}
\hline PD target Symptoms & H/V GAMES-related evidence based on SGs \\
\hline $\begin{array}{l}\text { Micrographia [30], } \\
\text { [67] }\end{array}$ & $\begin{array}{l}\text { SGs have focused on predictive PD analytics through pad games (e.g., a virtual farming theme game where the user is asked to perform } \\
\text { some tasks like cleaning the weeds following the trails shown on the screen by using two fingers) based on the data collected regarding } \\
\text { the trails of the player's fingers sliding on the screen [40]. Moreover, through this game, real-time data analysis is performed and alerts } \\
\text { can be sent to the doctors in case of detection of high risk of having PD. }\end{array}$ \\
\hline $\begin{array}{l}\text { Dysarthria [35], } \\
\text { Dysphonia [68] }\end{array}$ & $\begin{array}{l}\text { reach a high score. Study results indicated an increased peak voice loudness of the players' voice when playing the game. Digital games } \\
\text { can be one way to enhance patient's motivation for repetitive exercises both in therapy sessions and in prolonged use at home [69]. In } \\
\text { the work of [54], the design and implementation of a rehabilitation software (SG) for dysphonic patients was proposed, exploring the } \\
\text { extraction of pitch (Mel) feature from patients' voice for evaluating the long-time rehabilitation progress. }\end{array}$ \\
\hline \hline
\end{tabular}

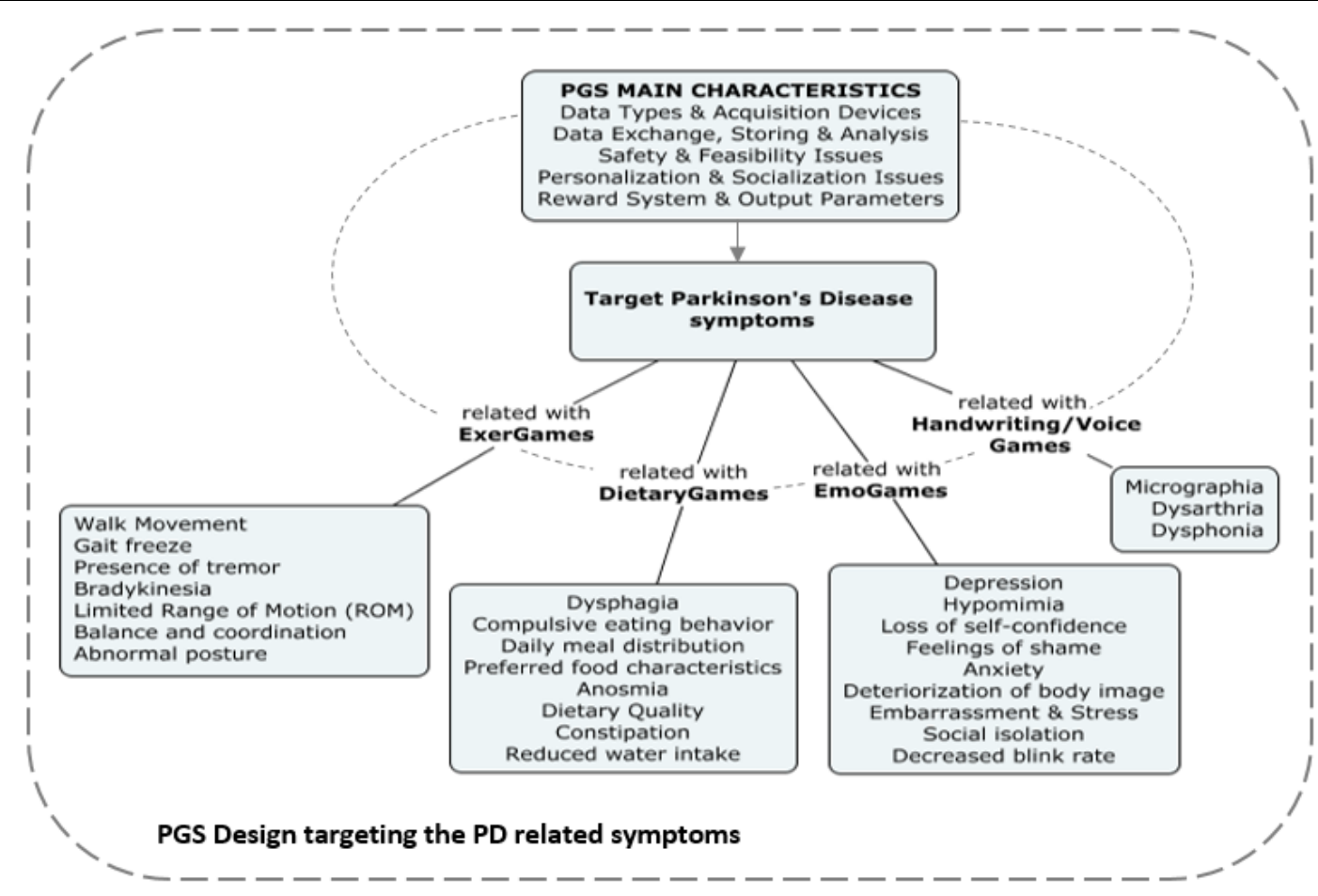

Figure 1. The i-PROGNOSIS Personalized Game Suite (PGS) design targeting the PD related symptoms.

\section{THE PROPOSED PGS DESIGN}

\section{A. PGS: General Framework}

As a first step, the i-PROGNOSIS PGS concept was defined, reflecting a common framework to be followed for each game design, based on five key transversal aspects (Fig. 1-top), namely: 1) Data types and acquisition devices (e.g., Kinect will be the main device for the ExerGames, while touch screens and tablets will be used by the PGS in a common way); 2) Data exchange, storing and analysis (e.g., in-game metrics will be analyzed in a common way, for instance: deviation, how many times a game was played); 3) Safety and feasibility issues (e.g., the PGS will constantly provide instructions, in order to avoid injuries); 4) Personalization and socialization issues (e.g., the games will be adjusted to the patients' performance and at the same time, the patient will participate in groups, for promoting collaboration/competition among the patients); and 5) Reward system and output parameters (e.g., the reward system will be applied to increase the engagement of the users in the game, by including motivational messages). More details are described in work of [70], as well as the specification of 14 game-scenarios/storyboards that were constructed based on the PGS general framework (Fig. 1-top).

Overall, taking into account the early stage PD patients' needs and requirements, the PGS aims to integrate different SGs in a unified platform [71], [72]. As seen in Fig.1-bottom, the target PD motor and non-motor symptoms are connected with the corresponding game (i.e., ExerGames, DietaryGames, EmoGames, and Handwriting/Voice Games), reflecting a common perspective and interdependence of the different SGs based on PGS main characteristics that are considered to all games. The provisional PGS design approach for each game is presented in detail in the next subsections.

\section{B. PGS: ExerGames Approach}

Regarding the PGS ExerGames approach: Gait features will be extracted by relevant ExerGames that request the user to move a couple of steps (within the Kinect range) towards different directions. The gait analysis from the games will be combined with the continuous balance assessment of the games based on the application of the Statically Equivalent Serial Chain (SESC) modeling [73] to the Kinect model, in order to 
utilize the center of mass component during walking. Body and hand motion analysis will evaluate whether Kinect (mainly used in the ExerGames) can detect tremor or not. The games will make the patients reaching large amplitude movements progressively, tracking the patients' performance (e.g., maximum angle) and setting an intermediate goal just above the patients' average performance. The ExerGames will continuously calculate the user's range of motion by introducing as in-game metrics the maximum body angles. The latter, will give more insights regarding the range of motion when the game instructions ask the user to take specific postures and gestures. Moreover, gait, balance and coordination features will arise from in-game metrics and mechanisms of the relevant ExerGames.

\section{PGS: DietaryGames Approach}

Concerning the PGS DietaryGames approach: a Mandometer will be used to measure the meal mechanics, representing a weighting scale that counts the progressive weight changes from a plate with food during a meal. The cumulative FI $\mathrm{w}(\mathrm{t})$ of the subject can be modelled using a quadratic curve $\mathrm{w}(\mathrm{t})=\alpha \mathrm{t} 2+\beta \mathrm{t}$, where $\alpha$ is the FI acceleration and $\beta$ is the initial FI rate. A way of defining meals is by nutritionally distinguishing meals from eating events: "meals refers to either breakfast, lunch or dinner, and snacks refer to all other eating/drinking events" [74]. Although the frequency and distribution of intake vary greatly across countries, meal categorization into breakfast, lunch and dinner are usually based on what time eating events are reported to be eaten. In general, the meal time distribution will be based on the following six distinct meals: breakfast, mid-morning snack, lunch, mid-afternoon snack, dinner and bedtime snack. In addition, during the design/developing of the PGS, a valuable approach based on the improvement of users' dietary habits will be considered to facilitate the choice of "healthy" foods instead of "unhealthy" foods. Moreover, a new way of modeling the food groups interconnection within the Eatwell Plate, combined with the water and emotional state, for the healthy ageing population was explored by [75]. From the methodological point of view, a Fuzzy-Logic (FL)-based approach was proposed as a means to provide a generalized measure of the Quality of Nutrition (QoN). The suggested FCM-QoN model has the potential to surface possibilities and scenarios from different perspectives, helping nutritionists/dietitians to promote a healthy lifestyle, assisting older adults to make better decisions regarding food choices as part of a healthy diet. The DietaryGames can easily follow this approach, since it has the potential to explore different dietary scenarios, helping health professionals to provide prognostic simulations for diseases effect (such as PD) on dietary habits.

\section{PGS: EmoGames Approach}

Concerning the PGS EmoGames approach: the sentiment monitoring from texts (e.g., SMS messages, tweets) created by a user will be used as a means for detecting depression. An $80 \%$ accuracy of this task has been reported [76]. From this perspective, new ways to detect motor impairments in facial muscles by analyzing selfies photos and/or videos that are captured by the user using his/her smartphone (e.g., while watching a short video/movie) will be considered in the design/development of the EmoGames approach. For instance, hypomimia detection methods, such as the ones explored in the work of [28], may be extended to use videos from standard cameras (e.g., mobile phone) instead of a 3-D camera sensor or other more obtrusive sensors.

\section{E. PGS: H/V Games Approach}

Regarding the PGS H/V Games approach, handwriting patterns could be acquired by either using of a graphical tablet or a stylus or by evaluating finger movement on a tablet computer or smartphone. The H/VGames will target on enhancing the handwriting patterns of early $\mathrm{PD}$ patients by prompting to write/draw specific letters and numbers with guidance lines and within specific space limits while providing real-time feedback of the performance. The H/VGames will be adaptable to the players' own writing styles and profiles. The $\mathrm{H} / \mathrm{V}$ Games will adopt and further enhance the existing approaches by incorporating the concept of handwriting patent correction and reestablishment. The captured data drawn by the user will be compared against the previous user's handwriting datasets, in order to assess the PD evolution, as well as to guide the game to adapt to the new conditions by adapting the guidance shapes and letters, providing real-time feedback. Grids and lines will appear and adapt correspondingly to the user's writing profile and micrographia level. Voice is captured by the tablet's or smartphone's microphone. Features like volume, loudness, pitch, formant frequencies will be extracted by signal processing algorithms from the patient's voice. Voice and breathing training games prompting the player to pronounce specific vowels/words at constant or alternating voice intensity levels, use shorter sentences and train vocal cords will be developed towards hypophonia limitation. Voice training games, along with breathing exercises, will constitute the voice intervention games. The $\mathrm{H} / \mathrm{V}$ Games will be combined with the EmoGames in the sense that the user will be requested to write the answer on the tablet or say it (when applicable), instead of just selecting an option by tapping on it. In this case, the user does not perceive this procedure as an intervention for her/his "problem", but as part of the games (which will be less stressful). Finally, the H/V Games will enrich the data with their in-game metrics, in order to serve as a "software as a sensor", towards early PD detection by multimodal monitoring.

\section{DISCUSSION}

The evaluation of the PGS relates with three pillars, i.e., the medical, technical and user acceptance evaluation. In particular, the most common rating scale used by medical professionals to assess a PD patients is the well-known Unified Parkinson's Disease Rating Scale (UPDRS) [77], essentially based on four dimensions, namely: mentation, behavior and mood; activities of daily living; motor examination and complications of therapy. In UPDRS, overall, a total of 42 features are examined, to assess the severity of the condition of each patient on a 0-4 scale (with the lower limit representing no physical or cognitive impairment, and the upper representing severe disability).

From the medical perspective, the effect of the PGS use on the PD patient's condition will be evaluated via the following 
medical tests per game category, periodically realized in a hospital setting:

- ExerGames

Gait mechanics: UPDRS; 6-minute walking test (6MWT); Timed up and Go test; 50-foot speed walk test; Dynamic Gait Index (DGI); Tremor: UPDRS; Bradykinesia: UPDRS; Limited Range of Motion: UPDRS; Balance and Coordination issues: Berg Balance Scale; Activitiesspecific Balance Confidence (ABC) Scale; Unipedal stance (eyes open-eyes close); Timed up and Go test; Dynamic Gait Index (DGI); Abnormal posture: UPDRS.

- DietaryGames

Meal mechanics: UPDRS, NMSS; Daily meal distributions: None - no test included till now, however, for ICD screening, the QUIP can be included, and for general daily meal distribution, a question in the part history taking can also be included. In addition, the NMSS can be used to identify general symptoms of swallowing problems and nausea and constipation issues. As an alternative, a new questionnaire regarding these aspects can also be constructed.; Preferred food characteristics and Dietary Quality: baseline characteristics - Habits (e.g., questioning regarding smoking, drinking alcohol, drinking caffeine containing beverages, chocolate consumption, red vegetables intakes), NMSS 20, 29, body weight and height.

- EmoGames

Depression: Parkinson's Disease Questionnaire QoL questionnaire (PDQ-39), Geriatric Depression Scale (GDS); Beck Depression Inventory (BDI); Hypomimia: Sub-item 19 of the UPDRS-motor score (UPDRS-III), which accounts only for the motor facet of hypomimia and gives no attention to the emotional aspect of facial expression.

- $\mathrm{H} / \mathrm{V}$ Games

Handwriting mechanics: writing sentences; Voice mechanics: UPDRS part II question 5, UPDRS part III question 18, Voice Handicap Index.

From the technical evaluation point of view, the PGS will be validated via the dynamic testing approach [78]. The later involves a set of test cases that take place when the code is executed. Moreover, dynamic testing may begin before the completion of the entire code of the product, in order to test particular sections, discrete functions and components, enabling the opportunity to identify early defects in the development process. In addition, an alternative procedure will be adopted, i.e., evolutionary approach, realized stepwise: i) a small piece or component (unit) of the modular product is developed, ii) the unit is tested, iii) in case of defects, the unit is repaired, iv) a new unit that adds functionality is developed, and v) the units are tested as an integrated component. The major advantage of this approach is that constantly, a working and useful product is delivered. Additionally, the testing is less complex and the errors are more easily identified and isolated.

Finally, the user acceptance evaluation will be based on a methodology grounded on the technology acceptance model (TAM) [79]. Data will be gathered via questionnaires and focus groups in order to monitor four main variables, i.e., facilitators, subjective norms, perceived usefulness and compatibility, that will enable the verification of the user friendliness of the system and the identification of potential users' intention to adopt i-PROGNOSIS system.

\section{CONCLUSIONS}

For each targeted PD symptom described in the previous sections, the i-PROGNOSIS PGS design approach intends not just to extend the UPDRS to all games, but also to monitor and support older adult's physical and emotional status enhancement, towards the decrease of the PD-related risks and increase of their QALYs, in a holistic and unobtrusive way. Via the i-PROGNOSIS PGS design approach, the selfmanagement of the PD patient's condition is placed within a serious games context, in order to improve, sustain or slowing down its progressive deterioration, considering safety, feasibility, personalization, socialization, and behavioural change issues. In the forthcoming period, a feedback collection period from the PD patients' and medical experts' will take place, in order to involve them as co-creators of the PGS gamedesign. This could increase the efficiency of the outputted game platform and could establish a bed-set for continuous improvement and add-on features, based on the user requirements and needs.

\section{ACKNOWLEDGMENT}

This work has received funding from the EU H2020-PHC2015, grant agreement $\mathrm{N}^{\circ}$ 690494: 'i-PROGNOSIS' project (www.i-prognosis.eu). The first author has been supported by the Foundation for Science and Technology (FCT, Portugal) (Postdoctoral Grant SFRH/BPD/496004/2013).

\section{REFERENCES}

[1] N. A. Bartolome, A. M. Zorrilla, and B. G. Zapirain, "Can game-based therapies be trusted? Is game-based education effective? A systematic review of the Serious Games for health and education," Proc. of the 16th International Conference on Computer Games (CGAMES), vol. 3, 2011, pp. 275-282.

[2] L. M. Reynolds, P. Hodge, and A. Simpson, "Serious games for mental health," Journal of Psychiatric and Mental Health Nursing, vol. 24(4), 2017, pp. 183-184.

[3] S. Göbel, and R. Maddison, "Serious Games for Health: The Potential of Metadata," Games for Health Journal, vol. 6(1), 2017, pp. 49-56.

[4] E. Dove, and A. J. Astell, "The use of motion-based technology for people living with dementia or mild cognitive impairment: A literature review," Journal of Medical Internet Research, vol. 19(1), 2017, e3, doi:10.2196/jmir.6518

[5] V. Vallejo, P. Wyss, A. Chesham, A. V. Mitache, R. M. Müri, U. P. Mosimann, and T. Nef, "Evaluation of a new serious game based multitasking assessment tool for cognition and activities of daily living: Comparison with a real cooking task," Computers in Human Behavior, vol. 70, 2017, pp. 500-506.

[6] H. F. Shu, T. Yang, S. X. Yu, H. D. Huang, L. L. Jiang, J. W. Gu, and Y. Q. Kuang, “Aerobic exercise for Parkinson's disease: A systematic review and meta-analysis of randomized controlled trials," PLoS One, vol. 9(7), 2014, e100503.

[7] G. Frazzitta, R. Maestri, D. Uccellini, G. Bertotti, and P. Abelli, "Rehabilitation treatment of gait in patients with parkinson's disease with freezing: A comparison between two physical therapy protocols using visual and auditory cues with or without treadmill training," Movement Disorders, vol. 24, pp. 1139-1143, 2009.

[8] S. Rahman, H. J. Griffin, N. P. Quinn, and M. Jahanshahi, "The factors that induce or overcome freezing of gait in Parkinson's disease," 
Behavioural Neurology, vol. 19(3), 2008, pp. 127-136, http://doi.org/10.1155/2008/456298

[9] D. M. Corcos, J. A. Robichaud, F. J. David, S. E. Leurgans, D. E. Vaillancourt, C. Poon, M. R. Rafferty, W. M. Kohrt, and C. L. Comella, "A two-year randomized controlled trial of progressive resistance exercise for Parkinson's disease," Movement Disorders, vol. 28(9), 2013, pp.1230-1240.

[10] D. Lilla, M. Herrlich, R. Malaka, and D. Krannich, "The influence of music on player performance in exergames for Parkinson's patients," Proc. of the International Conference on Entertainment Computing, Springer Berlin Heidelberg, 2012, pp. 433-436.

[11] S. Szlufik, M. Szumilas, J., Dutkiewicz, D. Koziorowski, T. Mandat, and E. Slubowska, "The impact of STN DBS on kinetic tremor in Parkinson's disease patients," Parkinsonism \& Related Disorders, vol. 22, 2016, p.e24.

[12] B. G. Farley, and G. F. Koshland, "Training BIG to move faster: the application of the speed-amplitude relation as a rehabilitation strategy for people with parkinson's disease," Experimental Brain Research, vol. 167(3), pp. 462-467, 2005

[13] L. T. Gobbi, M. D. Oliveira-Ferreira, M. J. D. Caetano, E. Lirani-Silva, F. A. Barbieri, F. Stella, and S. Gobbi, "Exercise programs improve mobility and balance in people with Parkinson's disease," Parkinsonism \& Related Disorders, vol. 15, 2009, S49-S52.

[14] G. Wulf, M. Landers, R. Lewthwaite, and T. Töllner, "External focus instructions reduce postural instability in individuals with Parkinson disease," Physical Therapy, vol. 89(2), 2009, pp. 162-168.

[15] M. E., Hackney, and G. M. Earhart, "Tai Chi improves balance and mobility in people with Parkinson disease," Gait \& Posture, vol. 28(3), 2008, 456-460, http://doi.org/10.1016/j.gaitpost.2008.02.005

[16] M. Ni, J. F. Signorile, K. Mooney, A. Balachandran, M. Potiaumpai, C. Luca, and J. G. Moore, "Comparative effect of power training and highspeed yoga on motor function in older patients with Parkinson disease," Arch of Physical Medicine and Rehabil., vol. 97(3), 2016, pp. 345-354.

[17] N. Argolo, M. Sampaio, P. Pinho, A. Melo, and A. C. Nóbrega, "Do swallowing exercises improve swallowing dynamic and quality of life in Parkinson's disease?," NeuroRehabilitation, vol. 32(4), 2013, pp. 949955, http://doi.org/10.3233/NRE-130918

[18] A. R. South, S. M. Somers, and M. S. Jog, "Gum chewing improves swallow frequency and latency in Parkinson patients: A preliminary study," Neurology, vol. 74(15), 2010, pp. 1198-202, doi 10.1212/WNL.0b013e3181d9002b.

[19] G. K. Pot, S. Almoosawi, and A. M. Stephen, "Meal irregularity and cardiometabolic consequences: Results from observational and intervention studies," Proceedings of the Nutrition Society, vol. 75(4), 2016, pp. 475-486, http://doi.org/10.1017/S0029665116000239

[20] M. Zandian, I. Ioakimidis, C. Bergh, and P. Södersten, "Linear eaters turned decelerated: Reduction of a risk for disordered eating?," Physiology \& Behavior, vol. 96(4-5), 2009, pp. 518-521, http://doi.org/10.1016/j.physbeh.2008.11.017

[21] I. Ioakimidis, M. Zandian, C. Bergh, and P. Södersten, "A method for the control of eating rate: a potential intervention in eating disorders," Behavior Research Methods, vol. 41(3), 2009, pp. 755-760, http://doi.org/10.3758/BRM.41.3.755

[22] V. Papapanagiotou, C. Diou, B. Langlet, I. Ioakimidis, and A. Delopoulos, "Automated Extraction of Food Intake Indicators from Continuous Meal Weight Measurements," Proc. of the International Conference on Bioinformatics and Biomedical Engineering, Springer International Publishing, 2015, pp. 35-46.

[23] C. Féart, C. Samieri, V. Rondeau, H. Amieva, F. Portet, J. F. Dartigues, N. Scarmeas, and P. Barberger-Gateau, "Adherence to a Mediterranean diet, cognitive decline, and risk of dementia," JAMA, vol. 302(6), 2009, pp.638-648

[24] F. Sofi, F. Cesari, R. Abbate, G. F. Gensini, and A. Casini, "Adherence to Mediterranean diet and health status: Meta-analysis," BMJ (Clinical Research Ed.), vol. 337, 2008, a1344, doi: https://doi.org/10.1136/bmj.a1344

[25] X. Gao, H. Chen, T. T. Fung, G. Logroscino, M. A. Schwarzschild, F. B. $\mathrm{Hu}$, and A. Ascherio, "Prospective study of dietary pattern and risk of
Parkinson disease," The American Journal of Clinical Nutrition, vol. 86(5), 2007, pp. 1486-1494.

[26] R. N. Alcalay, Y. Gu, H. Mejia-Santana, L. Cote, K. S. Marder, and N. Scarmeas, "The association between Mediterranean diet adherence and Parkinson's disease," Movement Disord., vol. 27(6), 2012, pp. 771-774.

[27] S. Chaffar, and D. Inkpen, "Using a heterogeneous dataset for emotion analysis in text," Proc. of the Canadian Conference on Artificial Intelligence, Springer Berlin Heidelberg, 2011, pp. 62-67.

[28] N. Vinokurov, D. Arkadir, E. Linetsky, H. Bergman, and D. Weinshall, "Quantifying hypomimia in parkinson patients using a depth camera," Proc. of the International Symposium on Pervasive Computing Paradigms for Mental Health, Springer, 2015, pp. 63-71.

[29] R. Mergl, P. Mavrogiorgou, U. Hegerl, and G. Juckel, "Kinematical analysis of emotionally induced facial expressions: A novel tool to investigate hypomimia in patients suffering from depression," Journal of Neurology, Neurosurgery \& Psychiatry, vol. 76(1), 2015, pp. 138-140.

[30] P. Drotar, J. Mekyska, Z. Smekal, I. Rektorova, L. Masarova, and M. Faundez-Zanuy, "Prediction potential of different handwriting tasks for diagnosis of Parkinson's," Proc. of the E-Health and Bioengineering Conference (EHB), IEEE, 2013, pp. 1-4

[31] N. Zhi, B. K. Jaeger, and A. Gouldstone, "Quantitative assessment of a therapeutic exercise in mitigating micrographia associated with Parkinson's Disease," Proc. of the Health Innovation and Point-of-Care Technologies Conference, Seattle, 2014, pp. 327-330.

[32] M. S. Bryant, D. H. Rintala, E. C. Lai, and E. C. Protas, "An investigation of two interventions for micrographia in individuals with Parkinson's disease," Clinical Rehabil., vol. 24, 2010, pp. 1021-1026

[33] R. M. Oliveira, J. M. Gurd, P. Nixon, J. C. Marshall, R. E. Passingham, "Micrographia in Parkinson's disease: The effect of providing external cues," Journal of Neurology, Neurosurgery and Psychiatry, vol. 63(4), 1997, pp. 429-433.

[34] L. O. Ramig, S. Sapir, S. Countryman, A. A. Pawlas, C. O'brien, M. Hoehn, and L. L. Thompson, "Intensive voice treatment (LSVT®) for patients with Parkinson's disease: A 2 year follow up," Journal of Neurology, Neurosurgery \& Psychiatry, vol. 71(4), 2001, pp. 493-498.

[35] J. Jankovic, "Parkinson's disease: clinical features and diagnosis," Journal of Neurology, Neurosurgery \& Psychiatry, vol. 79(4), 2008, pp. 368-376.

[36] M. S. Ekker, S. Janssen, J. Nonnekes, B. R. Bloem, and N. M. de Vries, "Neurorehabilitation for Parkinson's disease: Future perspectives for behavioural adaptation," Parkinsonism \& Related Disorders, vol. 22, 2016, S73-S77.

[37] O. Assad, R. Hermann, D. Lilla, B. Mellies, R. Meyer, L. Shevach, S. Siegel, M. Springer, S. Tiemkeo, J. Voges, and J. Wieferich, "Motionbased games for Parkinson's disease patients," Proc. of the International Conference on Entertainment Computing, Springer Berlin Heidelberg, 2011, pp. 47-58

[38] G. Barry, B. Galna, and L. Rochester, "The role of exergaming in Parkinson's disease rehabilitation: A systematic review of the evidence," Journal of Neuroengineering and Rehabilitation, 2014, doi: 10.1186/1743-0003-11-60.

[39] M. Hallett, and S. Khoshbin, "A physiological mechanism of bradykinesia," Brain, vol. 103(2), 1980, pp. 301-314

[40] S. Liu, Z. Shen, J. Mei, J. Ji, and C. Miao, "Parkinson's disease predictive analytics through a pad game based on personal data," International Journal of Information Techn., vol. 19(2), 2013, pp. 1-17.

[41] W. Yu, C. Vuong, and T. Ingalls, "An interactive multimedia system for Parkinson's patient rehabilitation," Proc. of the International Conference on Virtual and Mixed Reality, Springer Berlin Heidelberg, 2011, pp. 129-137.

[42] I. T. Paraskevopoulos, E. Tsekleves, C. Craig, C. Whyatt, and J. Cosmas, "Design guidelines for developing customised serious games for Parkinson's disease rehabilitation using bespoke game sensors," Entertainment Computing, vol. 5(4), 2014, pp. 413-424.

[43] M. L. Schenkman, K. Clark, T. Xie, and M. Kuchibhatla, "Spinal movement and performance of a standing reach task in participants with and without Parkinson's disease," Physical therapy, vol. 81(8), 2001, 1400-1411. 
[44] S. Siegel, and J. Smeddinck, "Adaptive difficulty with dynamic range of motion adjustments in exergames for Parkinson's disease patients," Proc. of the International Conference on Entertainment Computing, Springer Berlin Heidelberg, 2012, pp. 429-432.

[45] M. Plotnik, N. Giladi and J. M. Hausdorff, "Bilateral coordination of gait and Parkinson's disease: The effects of dual tasking," Journal of Neurology, Neurosurgery \& Psychiatry, vol. 80(3), 2009, pp. 347-350.

[46] R. McNaney, M. Balaam, A. Holden, G. Schofield, D. Jackson, M. Webster, B. Galna, G. Barry, L. Rochester, and P. Olivier, "Designing for and with people with parkinson's: A focus on exergaming," Proc. of the 33rd annual ACM conference on Human Factors in Computing Systems, ACM, 2015, pp. 501-510.

[47] P. V. Mhatre, I. Vilares, S. M. Stibb, M. V. Albert, L. Pickering, C. M. Marciniak, K. Kording, and S. Toledo, "Wii Fit balance board playing improves balance and gait in Parkinson's disease," PM\&R, vol. 5(9), 2013, pp. 769-777.

[48] J. E. Pompeu, F. A. dos Santos Mendes, K. G. da Silva, A. M. Lobo, T. de Paula Oliveira, A. P. Zomignani, and M. E. P. Piemonte, "Effect of Nintendo WiiTM-based motor and cognitive training on activities of daily living in patients with Parkinson's disease: A randomised clinical trial. Physiotherapy," vol. 98(3), 2012, pp. 196-204.

[49] F. B. Horak, J. G., Nutt, and L. M. Nashner, "Postural inflexibility in parkinsonian subjects," Journal of the Neurological Sciences, vol. 111, 1992, pp. 46-58

[50] Y. Okada, T. Shibata, T. Tamei, Y. Orito, H. Funaya, C. Obayashi, K. Ikeda, M. Hiyamizu, and S. Morioka, "In-home posture evaluation and visual feedback training to improve posture with a kinect-based system in Parkinson's disease: A case study," Movement Disorders, vol. 29, 256, 2014.

[51] G. Abbruzzese, R. Marchese, L. Avanzino, and E. Pelosin "Rehabilitation for Parkinson's disease: Current outlook and future challenges," Parkinsonism \& Related Dis., vol. 22, 2016, pp. S60-S64.

[52] I. Pachoulakis, and N. Papadopoulos, "Exergames for Parkinson's Disease patients: The balloon goon game," Proc. of the International Conference on Telecommunications and Multimedia (TEMU), IEEE, 2016, pp. 1-6.

[53] N. Miller, L. Allcock, A. J. Hildreth, D. Jones, E. Noble, and D. J. Burn, "Swallowing problems in Parkinson disease: Frequency and clinical correlates," Journal of Neurology, Neurosurgery, and Psychiatry, vol. 80(9), 2009, pp. 1047-1049, http://doi.org/10.1136/jnnp.2008.157701

[54] Z. Lv, C. Esteve, J. Chirivella, and P. Gagliardo, "A game based assistive tool for rehabilitation of dysphonic patients," Proc. of the 3rd IEEE VR international workshop on Virtual and augmented assistive technology (VAAT), IEEE, 2015, pp. 9-14.

[55] H. Miwa, and T. Kondo, "Alteration of eating behaviors in patients with Parkinson's disease: possibly overlooked?” Neurocase, vol. 14(6), 2008, pp. 480-484, http://doi.org/10.1080/13554790802495324

[56] T. Blackburne, A. Rodriguez, and S. J. Johnstone, "A Serious game to increase healthy food consumption in overweight or obese adults: Randomized controlled trial," JMIR Serious Games, vol. 4(2), 2016, doi: 10.2196/games.5708.

[57] B. Lorefält, A.-K. Granérus, and M. Unosson, "Avoidance of solid food in weight losing older patients with Parkinson's disease," Journal of Clinical Nursing, vol. 15(11), 2006, vol. 1404-1412, http://doi.org/10.1111/j.1365-2702.2005.01454.x

[58] J. A. Santiago, H. Bilyk, and J. A. Potashkin, "Understanding the role diet plays in Parkinson's disease could lead to better disease management," Clin Exp Pharmacol, vol. 5, 2014.

[59] A. Ueki, and M. Otsuka, "Life style risks of Parkinson's disease: Association between decreased water intake and constipation," Journal of Neurology, vol. 251(7), 2004, vii18-vii23.

[60] M. Rabbi, A. Pfammatter, M. Zhang, B. Spring, and T. Choudhury, "Automated personalized feedback for physical activity and dietary behavior change with mobile phones: A randomized controlled trial on adults," JMIR mHealth and uHealth, vol. 3(2), 2015 e42,doi: 10.2196/mhealth.4160

[61] Y. T. C. Yang, C. J. Wang, M. F. Tsai, and J. S. Wang, "Technologyenhanced game-based team learning for improving intake of food groups and nutritional elements," Computers \& Education, vol. 88, 2015, pp. 143-159.

[62] S. Paulmann, and M. D. Pell, "Dynamic emotion processing in Parkinson's disease as a function of channel availability," Journal of Clinical and Experimental Neuropsychology, vol. 32(8), 2010, pp. 822 835 .

[63] A. Anguenot, P. Y. Loll, J. P. Neau, P. Ingrand, and R. Gil, "Depression and Parkinson's disease: Study of a series of 135 Parkinson's patients," The Canadian Jour. of Neurological Sci., vol. 29(2), 2002, pp. 139-146.

[64] M. Springer, M. Herrlich, D. Krannich, and R. Malaka, "Achievements in exergames for Parkinson's patients,” In Mensch \& Computer, 2012, pp. 433-436.

[65] B. Galna, D. Jackson, G. Schofield, R. McNaney, M. Webster, G. Barry, D. Mhiripiri, M. Balaam, P. Olivier, and L. Rochester, "Retraining function in people with Parkinson's disease using the Microsoft kinect: Game design and pilot testing," Journal of Neuroengineering and Rehabilitation, vol. 11(60), 2014, doi: 10.1186/1743-0003-11-60

[66] M. M. Merzenich, and M. Nahum, U.S. Patent Application No. 15/017, 904, 2016.

[67] G. Gangadhar, D. Joseph, and V. Chakravarthy, "Understanding Parkinsonian handwriting through a computational model of basal ganglia," Neural Computation, vol. 20, 2008, pp. 2491-2528.

[68] S. Scott, and F. Caird, "Speech therapy for Parkinson's disease," Journal of Neurology, Neurosurgery \& Psychiatry, vol. 46, 1983, pp. 140-144.

[69] M. Krause, J. Smeddinck, and R. Meyer, "A digital game to support voice treatment for Parkinson's disease," Proc. of the CHI'13 extended abstracts on Human Factors in Computing Systems, ACM, 2013.

[70] S. B. Dias, E. Konstantinidis, J. A. Diniz, P. Bamidis, V. Charisis, S Hadjidimitriou, M. Stadtschnitzer, P. Fagerberg, I. Ioakeimidis, K. Dimitropoulos, N. Grammalidis, and L.J. Hadjileontiadis, "On supporting Parkinson's Disease patients: The i-PROGNOSIS Personalized Game Suite design approach," Proc. of the 30th IEEE International Symposium on Computer-Based Medical Systems, 2017.

[71] S. Hadjidimitriou, V. Charisis, K. Kyritsis, E. Konstantinidis, A. Delopoulos, P. Bamidis et al., "Active and healthy ageing for parkinson's disease patients' support: A user's perspective within the iPROGNOSIS framework," Proc. of the International Conference on Technology and Innovation in Sports, Health and Wellbeing (TISHW), IEEE, 2016, pp. 1-8.

[72] S. Dias, J. A. Diniz, S. Hadjidimitriou, V. Charisis, E. Konstantinidis, P D. Bamidis, and L. J. Hadjileontiadis, "Personalized Game Suite: A unified platform to sustain and improve the quality of life of parkinson's disease patients," Frontiers in Human Neuroscience, vol. 10(23), 2016.

[73] S. Cotton,A. Murray, and P. Fraisse, "Statically equivalent serial chains for modeling the center of mass of humanoid robots," Proc. of $8^{\text {th }}$ International Conference on Humanoid Robots (IEEE-RAS), IEEE, pp. 138-144.

[74] C. de Graaf, "Nutritional definitions of the meal," In H. L. Meiselman, Ed., Dimensions of the meal: The science, culture, business and art of eating, Gaithersburg, MD: Aspen, 2000, pp. 47-59.

[75] S. B. Dias, S. J. Hadjileontiadou, J. A. Diniz, J. Barroso, and L. J. Hadjileontiadis, "On modeling the quality of nutrition for healthy ageing using fuzzy cognitive maps,". In M. Antona and C. Stephanidis, Eds., Access in Human-Computer Interaction. Users and Context Diversity. LNCS, vol. 9739, Springer International Publishing, 2016, pp. 332-343.

[76] C. Wang, Z. Xiao, Y. Liu, Y. Xu, A. Zhou, and K. Zhang, "SentiView: Sentiment analysis and visualization for internet popular topics," IEEE Transactions on Human-Machine Syst., vol. 43(6), pp. 620-630, 2013.

[77] G. Ebersbach, H. Baas, I. Csoti, M. Müngersdorf, G. Deuschl, "Scales in Parkinson's Disease,” Journal of Neurology, vol. 253, suppl.4, pp. iv32iv35, doi:10.1007/s00415-006-4008-0

[78] L. Crispin, and J. Gregory, "Agile testing: A practical guide for testers and agile teams," Pearson Education, 2009.

[79] F. D. Davis, "User acceptance of information technology: System characteristics, user perceptions and behavioral impacts," Internationa Journal of Man-Machine Studies, vol. 38(3), 1993, pp. 475-487, http://hdl.handle.net/2027.42/30954 